\title{
Potential for the Repurposing of Adamantane Antivirals for COVID-19
}

\author{
Roger F. Butterworth ${ }^{1}$ (i)
}

Accepted: 20 May 2021 / Published online: 21 June 2021

(c) The Author(s) 2021

\begin{abstract}
Several adamantanes have established actions against coronaviruses. Amantadine, rimantadine, bananins and the structurally related memantine are effective against human respiratory coronavirus $\mathrm{HCoV-OC43,} \mathrm{bovine} \mathrm{coronavirus} \mathrm{and} \mathrm{severe} \mathrm{acute}$ respiratory syndrome coronavirus 1 (SARS-CoV-1) and a spiroadamantane amine is effective against the coronavirus strain 229E. Molecular docking studies suggest that amantadine may block the viral E protein channel, leading to impaired viral propagation. Additionally, amantadine analogues may inhibit entry of the virus into the host cell by increasing the $\mathrm{pH}$ of the endosomes and thus inhibiting the action of host cell proteases such as Cathepsin L. High-throughput drug screen gene expression analysis identified compounds able to down-regulate Cathepsin L expression where the fifth most potent agent of 466 candidates was amantadine. Amantadine inhibits severe acute respiratory syndrome coronavirus 2 replication in vitro but does not inhibit the binding of the spike protein to ACE2. Adamantanes also may act against coronaviruses including severe acute respiratory syndrome coronavirus 2 (SARS-CoV-2) via antagonism of glutamate (NMDA) and the $\alpha-7$ subtype of the nicotinic acetylcholine receptor located on bronchial and alveolar epithelial cells. As an NMDA receptor antagonist, memantine has the potential to inhibit entry of SARS-CoV-2 into these cell populations. Amantadine and memantine are widely employed for the treatment of neurodegenerative diseases and a pathophysiologic link between the antiviral and anti-Parkinson actions of amantadine has been entertained. Case reports involving 23 patients with reverse transcription polymerase chain reactionconfirmed coronavirus disease 2019 (COVID-19) and a range of co-morbidities including type 2 diabetes mellitus, Parkinson's disease, multiple sclerosis and severe cognitive impairment reveal significant potential benefits of amantadine and memantine for the prevention and/or treatment of coronavirus disease 2019 and its neurological complications.
\end{abstract}

\section{Introduction}

Serious attempts are currently ongoing for the repurposing of existing agents including antibiotics, antimalarials and antivirals for the treatment of COVID-19. With regard to antiviral agents in particular, several members of the adamantane family have well-established antiviral properties principally against a range of influenza viruses. Structures of common adamantane antivirals are shown in Fig. 1.

Examples of the antiviral actions of these adamantanes include blocking of the M2 proton channel of the influenza A virus by amantadine and rimantadine, resulting in inhibition of the endosomal uncoating of the virus $[1,2]$, while a novel spiroadamantane amine is also a highly potent inhibitor of the

Roger F. Butterworth

rb@enceph.com

1 Department of Medicine, University of Montreal, 45143

Cabot Trail, Englishtown, NS B0C 1H0, Canada amantadine-resistant V27A mutant [3] and a series of novel 2-aminoadamantanes are effective against a range of H1N1 strains of influenza A [4].

The aims of the present study were two-fold, namely, (1) the identification of published reports of preclinical and/or early clinical investigations in favour of the repurposing of members of the adamantane family of antiviral agents for COVID-19 and (2) insights into possible mechanisms of action of adamantanes in this regard.

Amantadine, rimantadine and the structurally related memantine (Fig. 1) are also effective against the coronaviruses including $\mathrm{HCoV}-\mathrm{OC} 43$, the bovine coronavirus [5] and SARS-CoV-1. Bananins, a class of antiviral compounds composed of a trioxa-adamantane moiety bound to a pyridoxal derivative, show efficacy against both $\mathrm{HCoV}-\mathrm{OC} 43$ and SARS-CoV-1 [6, 7] and spiroadamantane amine is effective against the coronavirus strain 229E [3].

These findings form the basis and rationale for the present review and have resulted in a call for the repurposing of adamantanes for COVID-19 on an expanded basis [7]. Electronic 


\section{Key Points}

Members of the adamantane family have established actions against coronaviruses.

Amantadine may block the viral E protein ion channel function and may down-regulate the protease Cathepsin $\mathrm{L}$ because of the increase of lysosomal $\mathrm{pH}$, resulting in impaired viral entry and replication.

Acting as antagonists of key excitatory and inhibitory neurotransmitters, amantadine and memantine have the capacity to mitigate the central nervous system complications of COVID-19 including the acute respiratory failure.

Initial results in small cohorts of patients or case reports reveal beneficial actions of amantadine or memantine in patients with COVID-19 even in the presence of comorbidities and a single case of COVID-19 prophylaxis by amantadine has been reported.

Confirmation and extension of these findings are now required in randomised controlled trials of candidate adamantane anti-viral agents.

and manual searches of databases were conducted including MEDLINE, PubMed and Google Search with appropriate keywords that included: adamantane(s), amantadine, rimantadine, memantine, coronavirus, SARS-CoV-1, SARS-CoV-2 and COVID-19 for the period 1973-2021. Searches were extended to include references cited in systematic reviews of pertinent topics published in the English, French or German languages and included editorials, book chapters, short communications and letters to journal editors.

\section{Adamantanes Against SARS-CoV-2: In Vitro and Preclinical Investigations}

A recent study tested the efficacy in a dose-response paradigm of amantadine in vitro on Vero E6 cells infected with SARS-CoV-2 [8]. Amantadine (0.3-300 $\mu \mathrm{M})$ did not inhibit the binding of the SARS-CoV-2 spike protein to the human ACE2 protein as previously suggested by docking calculations [9]. However, amantadine reproducibly inhibited viral replication with a half-maximal effective concentration $\left(\mathrm{EC}_{50}\right)$ range of $83-119 \mu \mathrm{M}$, which is somewhat higher than therapeutic concentrations after systemic administration.

Recent advances towards the elucidation of the molecular mechanisms implicated in the entry of SARS-CoV-2 into the host cell and the effects of amantadine on protein-protein interactions implicated in these mechanisms have resulted in the formulation of possible mechanisms. The SARS-CoV-2 virus contains four proteins: $\mathrm{E}$ and $\mathrm{M}$ proteins form part of the viral envelope; $\mathrm{N}$ protein binds to the viral genome; and the $\mathrm{S}$ (spike) protein binds to the host cell receptor ACE2. Molecular docking studies suggest that amantadine, via interactions with specific amino acids, has the potential to bind to the transmembrane domain of protein E (ETM) [10].

In a bid to identify underlying mechanisms and candidate drugs for COVID-19, an analysis of differentially expressed genes that co-express with ACE2 indicated by the expression of RNAs isolated from bronchoalveolar lavage fluid cells of patients with COVID-19 by functional enrichment and hub gene cluster analyses were undertaken [11]. Using the connectivity map database with transcriptome profiles of patients with COVID-19, candidate drugs were identified, one of which was amantadine.

These docking calculations suggested that an interaction occurs by hydrogen bridging of amantadine with amino acids PHE26 and ALA22, leading to impaired release of the virus into the host cell and inhibition of its propagation [10]. A bacterial assay suggests that the SARS-CoV-2 E protein may also be inhibited by memantine [12]. The issue relating to the importance of the $\mathrm{E}$ protein channel has recently been

Fig. 1 Structures of amantadine, rimantadine and the structurally related analogue memantine, which manifest significant actions against a range of coronaviruses

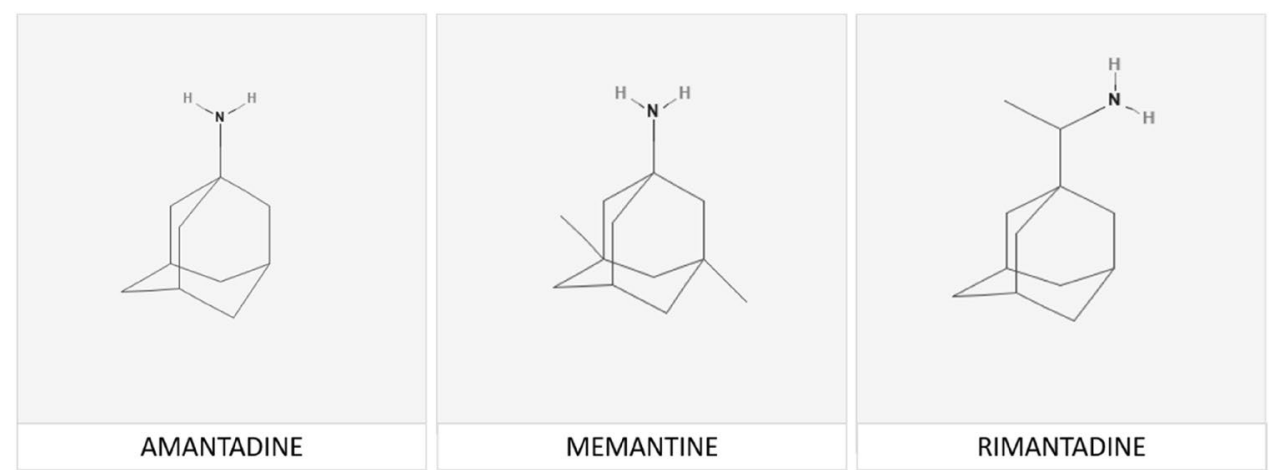


revisited following elucidation of the structure of the ETM from SARS-CoV-2 [13], which was found comparable [14] to that of SARS-CoV-1 in which amantadine significantly inhibited protein E ion channel-mediated activity [15].

A third potential mechanism focuses on the lysosomotropic action of amantadine [16, 17]. Cleavage of the S protein by host proteases is critical for viral activation and subsequent infection and, given the similarity to SARS-CoV-1, SARS-CoV-2 takes advantage of the endosomal proteases Cathepsin B and L (CTSL, CTSB).

Cathepsin L is a key element of the lysosomal pathway and its disruption is likely to provide a potential therapy for COVID-19. Mechanisms of disruption include decreased expression of the CTSL gene, inhibition of CTSL activity and alterations of its environment following, for example, an increase of lysosomal $\mathrm{pH}$. A simplified schematic of some of the key steps and associated proteins implicated in the invasion of the host cell by SARS CoV-2 is depicted in Fig. 2 where binding to ACE2 is followed by cleavage due to CTSL, resulting in fusion of viral and host cell membranes and release of the viral genome.

Circulating levels of CTSL are increased after SARSCoV-2 infection where they are positively correlated with disease course and severity [18] and, importantly, SARS-CoV-2 pseudovirus infection results in increased CTSL expression in human cells in vitro and in human ACE2-transgenic mice in vivo, while CTSL overexpression leads to enhancement of pseudovirus infection in human cells. Moreover, CTSL functionally cleaves the SARS-CoV-2 spike protein leading to enhanced viral entry and amantadine significantly inhibited
CTSL activity after SARS-CoV-2 pseudovirus infection both in vitro and in vivo.

In an independent series of investigations, high-throughput drug screen gene expression analysis identified compounds with the capacity to down-regulate CTSL expression in a well-characterized, retinal pigment epithelial cell line. One of the most potent agents for the down-regulation of the CTSL gene was amantadine $(10 \mu \mathrm{M})$, which ranked fifth of 466 compounds tested. Furthermore, the lysosomal trapping capacity of amantadine owing to its lipophilicity and increase of lysosomal $\mathrm{pH}$ may be indicative of interference with the capacity of the virus to replicate. Together, these findings suggest that amantadine has the potential to decrease the viral load in SARS-CoV-2-infected patients leading to decreased replication and infectivity of the virus [16].

Damage to the central nervous system (CNS) is well established in COVID-19 and it has been suggested that the neuroinvasive nature of SARS-CoV-2 may contribute to the acute respiratory failure characteristic of COVID-19 [19]. There is evidence to suggest that amantadine and memantine exert their protective effects against COVID-19 via mechanisms involving specific CNS neurotransmitter systems [20]. Both amantadine and memantine are potent antagonists of the NMDA subclass of glutamate receptor and memantine prevents motor incoordination problems in animals infected with the neuroinvasive $\mathrm{HCoV-OC43}$ with concomitant suppression of viral replication in a dose-dependent manner. Rimantadine has inhibitory effects on SARS-CoV-1 and both amantadine and rimantadine have the capacity to block viral entry into vulnerable cell populations. Memantine is
Fig. 2 Simplified schematic of key steps and associated proteins involved in the invasion of the host cell by SARS-CoV-2. Binding of the SARS-CoV-2 spike to ACE2 is followed by action of the endosomal cysteine protease Cathepsin L [CTSL], resulting in fusion of viral and host cell membranes and release of the viral genome into the host cell cytoplasm. Amantadine has the potential to disrupt the process by downregulation of CTSL by increasing the $\mathrm{pH}$ of endosomes, resulting in impaired viral entry and replication [20]

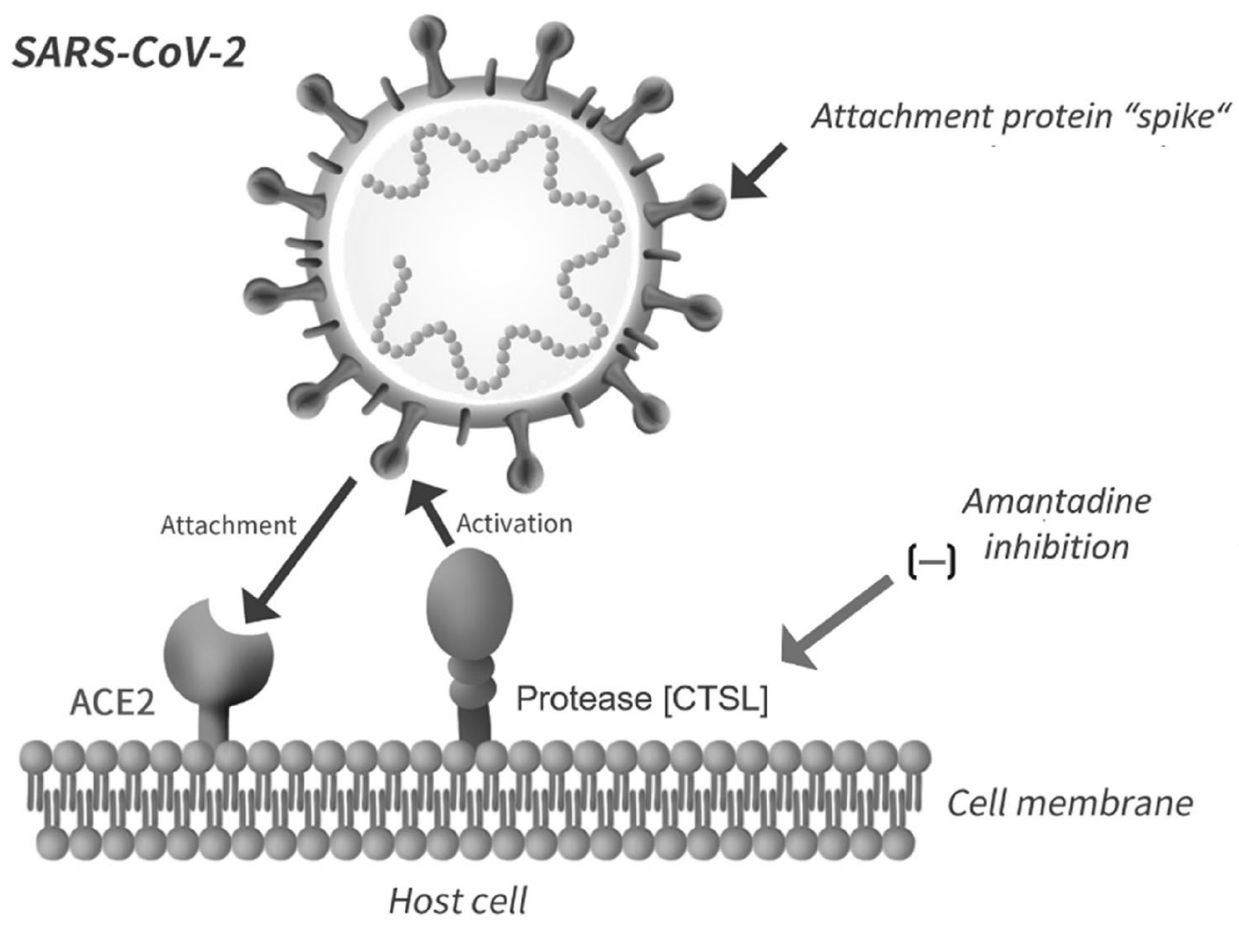


also a potent antagonist of the $\alpha-7$ subtype of the nicotinic acetylcholine receptor, leading to decreased ACE2 receptor expression in respiratory epithelial cells with the potential to inhibit the entry of SARS-CoV-2 [21].

\section{Adamantanes for the Prevention and Treatment of COVID-19: Initial Clinical Evidence}

Given the relatively short period of time during which the potential for re-purposing of adamantanes for COVID-19 has been under consideration, clinical evidence relies by necessity on findings from observational/uncontrolled studies and case reports.

In the first of two case reports, a 57-year-old man with type 2 diabetes of 10 years duration with cold symptoms, muscle pain and a persistent cough tested positive by reverse transcription-polymerase chain reaction for SARS-CoV-2 in a pharyngeal exudate sample [22]. His oxygen saturation was $84 \%$; he was given supplementary oxygen, prescribed amantadine (100 mg twice daily [bid]) and nebulized with ipratropium bromide/salbutamol $(0.5 \mathrm{mg}, 2.5 \mathrm{mg} / 2.5 \mathrm{~mL})$ for 5 days with the addition of $500 \mathrm{mg}$ of aspirin for 5 days. He continued metformin ( $850 \mathrm{mg}$ bid) for the treatment of his diabetes. The patient's wife (age 54 years) and daughter (age 33 years) tested positive for SARS-CoV-2, and, although asymptomatic, were prescribed amantadine (100 mg bid) for 14 days as a preventive measure. The male patient's oxygen saturation levels and clinical status improved gradually, leading to his release from hospital on day 14. Neither of his family members went on to develop symptoms of COVID-19.

In a second report, 22 patients with severe neurological disorders (five with Parkinson's disease [PD], ten with multiple sclerosis [MS] and seven with cognitive impairment) tested positive for SARS-CoV-2 by reverse transcriptionpolymerase chain reaction in nasopharyngeal swabs. All patients had been receiving adamantanes for at least 3 months prior to testing positive for COVID-19. In PD, amantadine is effective both for improvements in motor function and also for the treatment of L-Dopa-induced dyskinesias [23] and all patients with PD in the above case report had been prescribed L-Dopa in addition to amantadine $(100 \mathrm{mg} /$ day for $>3$ months prior to testing positive for COVID-19). None of the five amantadine-treated patients with PD developed clinical signs of infection (no fever, cough or anosmia) while control of extrapyramidal motor function was unaffected [24]. Interestingly, a recent review summarized possible pathophysiologic links between mechanisms of anti-SARSCoV-2 and anti-PD actions of amantadine [25].

In a second cohort, the ten patients with MS (age: $38 \pm 10$ years, three male individuals), who tested positive for COVID-19 by reverse transcription-polymerase chain reaction of nasopharyngeal swabs had been receiving amantadine (100 mg once daily) for the treatment of MS-related fatigue for at least 3 months prior to infection. Following documented exposure to the virus, a 14-day quarantine was observed in all cases. None of the ten cases developed clinical manifestations typical of COVID-19 [24]. This report prompted a retrospective review of memantine use and COVID-19-associated mortality from a South Korean national database in which 5726 patients with confirmed COVID-19 were identified, 140 of whom had died [26]. The relationship between treatment with memantine and COVID19-associated mortality was investigated. No statistically significant relationship between COVID-19-associated mortality and ongoing treatment with memantine after adjustment for clinically relevant potential confounders (age, sex, co-morbidities) was observed either in the analysis of all patients or in the sub-group of patients with dementia. It was proposed that future studies may wish to evaluate the effects of memantine on the clinical course of the disease or on clinical outcomes according to disease severity.

In the third group, seven patients (mean age: $71 \pm 10$ years, four male individuals) with cognitive impairment of $>7$ years duration who had been receiving memantine (10 $\mathrm{mg}$ bid) for a period in excess of 3 months tested positive for COVID19. A 14-day quarantine was observed in all cases following infection. Again, as with the PD and MS patient groups described above, none of the seven patients developed clinical symptoms of infectious disease [24].

\section{Potential Use of Adamantanes for the Prevention and Treatment of CNS Complications of COVID-19}

Widespread CNS damage and associated symptomatology due to SARS-CoV-2 viral infection are now well established; features include an impaired level and content of consciousness, impairments of taste, smell and vision, and neuromuscular disorders. Moreover, the neuroinvasive nature of SARS-CoV-2 probably contributes to the acute respiratory failure characteristic of COVID-19 [19]. Pathologically, infection with the coronavirus SARS-CoV-1 results in viral detection in the brains of infected patients accompanied by neuronal necrosis and glial hyperplasia with a pattern that is reminiscent of some aspects of traumatic brain injury [27]. Interestingly, amantadine is proven to be beneficial for the treatment of disorders of consciousness in the context of traumatic brain injury where the beneficial effect results from increased production of dopamin secondary to NMDA receptor antagonism [27]. Memantine, like amantadine, is an NMDA receptor antagonist and is neuroprotective against a range of neurodegenerative disorders and against the neuroinvasive $\mathrm{HCoV}-\mathrm{OC} 43$ in which it prevented neuronal cell 
damage and death with concomitant reduction in viral replication, all of which occurred in a dose-dependent manner [28].

\section{Summary and Future Directions}

In vitro and preclinical studies confirm that several members of the adamantane family of antiviral agents are effective against a range of coronaviruses including SARS-CoV-2, the virus responsible for COVID-19. The most likely candidates include amantadine, rimantadine, memantine and the bananins. Mechanisms whereby these agents exert their antiviral actions are somewhat dependent on the identity of both the coronavirus and the adamantane itself. Evidence to date suggests mechanisms relating to interference of the viroporin $\mathrm{E}$ channel of the virus, while it is likely that the down-regulation of essential host cell proteases necessary for viral replication is also implicated. With reference to the latter, it was noted that the mean effective dose $\left(E_{50}\right)$ for amantadine was within one order of magnitude of its pharmacokinetic profile with relatively mild side effects compared with other currently available, putative COVID-19 treatments [16]. Taken together, these findings constitute a good experimental evidence base and rationale for further study with a view to repurposing adamantane antiviral agents as antiSARS-CoV-2 agents.

Future research and development in this area has a unique opportunity to move in multiple directions. For example, there are currently more than 200 adamantane derivatives $[29,30]$ with distinct properties. Many have proven actions against a range of viruses including some coronaviruses, but few so far have been tested against SARS-CoV-2 even in the context of simple in vitro preparations. For example, where studies were made of in vitro susceptibility of ten clinical isolates of SARS-CoV-1 to selected antiviral compounds that included amantadine or rimantadine, significant inhibitory activities were confirmed for rimantadine $\left(\mathrm{EC}_{50}: 8-16 \mu \mathrm{g} /\right.$ $\mathrm{mL}$ ) but amantadine was inactive [31]. It is surprising in view of these findings that there has been minimal research and development relating to the use of rimantadine against SARS-CoV-1 or SARS-CoV-2 (assuming that ETM is the target of antiviral action and that the sequence of the two species is $100 \%$ identical) [15]. From a related perspective, it is not unlikely that future investigators with expertise in synthetic organic chemistry may wish to consider research targeting newly synthesized adamantane molecules with novel functional groups as potential antiviral agents for COVID-19 $[29,30]$.

The proposal that certain adamantanes (amantadine, memantine) may be of benefit for the treatment of neurological complications of COVID-19 by virtue of their established actions as mediators of key excitatory and inhibitory neurotransmitter systems [20] will likely lead to continued work in this area that could result in novel therapies for the characteristic CNS symptoms of "long COVID-19".

From the clinical perspective, initial results from small cohorts of patients or single case reports offer promise. In particular, reports alluding to the benefit of amantadine or memantine for COVID-19 treatment in patients with significant co-morbidities [24] are encouraging as is the single case of an apparent COVID-19 prophylaxis by amantadine [32]. Confirmation of these initial results is now required in adequately powered, randomized controlled clinical trials.

\section{Declarations}

Funding No sources of funding were received for the preparation of this article.

Conflicts of Interest/Competing Interests Roger F. Butterworth has received ad hoc payments for consulting and speaker fees from Salix Pharmaceuticals (USA), Umecrine Pharmaceuticals (Sweden) and Merz Pharmaceuticals (Germany).

Ethics Approval Not applicable.

Consent to Participate Not applicable.

Consent for Publication Not applicable.

Availability of Data and Material Not applicable.

Code Availability Not applicable.

Authors' Contributions Roger F. Butterworth was the sole author of this article.

Open Access This article is licensed under a Creative Commons Attribution-NonCommercial 4.0 International License, which permits any non-commercial use, sharing, adaptation, distribution and reproduction in any medium or format, as long as you give appropriate credit to the original author(s) and the source, provide a link to the Creative Commons licence, and indicate if changes were made. The images or other third party material in this article are included in the article's Creative Commons licence, unless indicated otherwise in a credit line to the material. If material is not included in the article's Creative Commons licence and your intended use is not permitted by statutory regulation or exceeds the permitted use, you will need to obtain permission directly from the copyright holder. To view a copy of this licence, visit http:// creativecommons.org/licenses/by-nc/4.0/.

\section{References}

1. Bukrinskaya AG, Vorkunova NK, Narmanbetova RA. Rimantadine hydrochloride blocks the second step of influenza virus uncoating. Arch Virol. 1980;66(3):275-82. https://doi.org/10.1007/BF013 14742 .

2. Balgi AD, Wang J, Cheng DY, Ma C, Pfeifer TA, Shimizu Y, et al. Inhibitors of the influenza A virus M2 proton channel discovered using a high-throughput yeast growth restoration assay. PLoS One. 
2013;8(2):e55271. https://doi.org/10.1371/journal.pone.0055271 (Epub 2020 Mar 16).

3. Mathur A, Beare AS, Reed SE. In vitro antiviral activity and preliminary clinical trials of a new adamantane compound. Antimicrob Agents Chemother. 1973;4(4):421-6. https://doi.org/10.1128/ aac.4.4.421.

4. Kolocouris A, Tzitzoglaki C, Johnson FB, Zell R, Wright AK, Cross TA, et al. Aminoadamantanes with persistent in vitro efficacy against H1N1 (2009) influenza A. J Med Chem. 2014;57(11):4629-39. https://doi.org/10.1021/jm500598u (Epub 2014 May 15).

5. Payne HR, Storz J, Henk WG. Initial events in bovine coronavirus infection: analysis through immunogold probes and lysosomotropic inhibitors. Arch Virol. 1990;114(3-4):175-89. https://doi.org/10. 1007/BF01310747.

6. Tanner JA, Zheng BJ, Zhou J, Watt RM, Jiang JQ, Wong KL, et al. The adamantane-derived bananins are potent inhibitors of the helicase activities and replication of SARS coronavirus. Chem Biol. 2005;12(3):303-11. https://doi.org/10.1016/j.chembiol.2005. 01.006 .

7. Cimolai N. Potentially repurposing adamantanes for COVID-19. J Med Virol. 2020;92(6):531-2. https://doi.org/10.1002/jmv.25752 (Epub 2020 Mar 16).

8. Fink K, Nitsche A, Neumann M, Grossegesse M, Eisele KH, Danysz W. Amantadine inhibits SARS-CoV-2 in vitro. Viruses. 2021;13(4):539. https://doi.org/10.3390/v13040539.

9. Baig AM, Khaleeq A, Syeda H. Docking prediction of amantadine in the receptor binding domain of spike protein of SARS-CoV-2. ACS Pharmacol Transl Sci. 2020;3(6):1430-3. https://doi.org/10. 1021/acsptsci.0c00172.

10. Abreu GEA, Aguilar MEH, Covarrubias DH, Durán FR. Amantadine as a drug to mitigate the effects of COVID-19. Med Hypotheses. 2020;140:109755. https://doi.org/10.1016/j.mehy.2020. 109755 (Epub 2020 Apr 25).

11. Li Z, Yang L. Underlying mechanisms and candidate drugs for COVID-19 based on the connectivity map database. Front Genet. 2020;27(11): https://doi.org/10.3389/fgene.2020.558557.

12. Singh Tomar PP, Arkin IT. SARS-CoV-2 E protein is a potential ion channel that can be inhibited by gliclazide and memantine. BiochemBiophys Res Commun. 2020;30(1):10-14. https://doi.org/ 10.1016/j.bbrc.2020.05.206 (Epub 2020 Jun 20).

13. Mandala VS, McKay MJ, Shcherbakov AA, Dregni AJ, Kolocouris A, Hong M. Structure and drug binding of the SARS-CoV-2 envelope protein transmembrane domain in lipid bilayers. Nat Struct Mol Biol. 2020;27(12):1202-8. https://doi.org/10.1038/s41594020-00536-8 (Epub 2020 Nov 11).

14. Surya W, Li Y, Torres J. Structural model of the SARS coronavirus E channel in LMPG micelles. Biochim Biophys Acta Biomembr. 2018;1860(6):1309-17. https://doi.org/10.1016/j.bbamem.2018. 02.017 (Epub 2018 Feb 21).

15. Jeppesen MG, Toft-Bertelsen TL, Kledal TN, Rosenkilde MM. Amantadin has potential for the treatment of COVID-19 because it targets known and novel ion channels encoded by SARS-CoV-2. Res Sq. 2021. https://doi.org/10.21203/rs.3.rs-121743/v1.

16. Smieszek SP, Przychodzen BP, Polymeropoulos MH. Amantadine disrupts lysosomal gene expression: a hypothesis for COVID19 treatment. Int J Antimicrob Agents. 2020;55(6):106004. https:// doi.org/10.1016/j.ijantimicag.2020.106004 (Epub 2020 Apr 30).

17. Tzitzoglaki C, McGuire K, Lagarias P, Konstantinidi A, Hoffmann A, Fokina NA, et al. Chemical probes for blocking of influenza A M2 wild-type and S31N channels. ACS Chem Biol. 2020;15(9):2331-7. https://doi.org/10.1021/acschembio.0c00553 (Epub 2020 Aug 27).
18. Zhao MM, Yang WL, Yang FY, Zhang L, Huang WJ, Hou W, et al. Cathepsin $\mathrm{L}$ plays a key role in SARS-CoV-2 infection in humans and humanized mice and is a promising target for new drug development. Signal Transduct Targ Ther. 2021;6(1):134. https://doi. org/10.1038/s41392-021-00558-8.

19. Li YC, Bai WZ, Hashikawa T. Response to commentary on "The neuroinvasive potential of SARS-CoV-2 may play a role in the respiratory failure of COVID-19 patients". J Med Virol. 2020;92(7):707-9. https://doi.org/10.1002/jmv.25824 (Epub 2020 Apr 10).

20. Brenner SR, Butterworth RF. Repurposing of adamantanes with transmitter receptor antagonist properties for the prevention/treatment of COVID-19. J Pharmaceu Pharmacol. 2020;8(1):4. https:// doi.org/10.13188/2327-204X.S200005.

21. Hasanagic S, Serdarevic F. Potential role of memantine in the prevention and treatment of COVID-19: its antagonism of nicotinic acetylcholine receptors and beyond. Eur Respir J. 2020;56(2):2001610. https://doi.org/10.1183/13993003. 01610-2020.

22. Aranda-Abreu GE, Aranda-Martinez JD, Araujo R. Use of amantadine in a patient with SARS-CoV-2. J Med Virol. 2021;93(1):110 1. https://doi.org/10.1002/jmv.26179.

23. Butterworth RF. Amantadine for the treatment of Parkinson's disease and its associated dyskinesias. J Parkinsons Dis Alzheimer Dis. 2020;7(1):7.

24. Rejdak K, Grieb P. Adamantanes might be protective from COVID-19 in patients with neurological diseases: multiple sclerosis, parkinsonism and cognitive impairment. Mult Scler Relat Disord. 2020;42:102163. https://doi.org/10.1016/j.msard.2020. 102163 (Epub 2020 Apr 30).

25. Butterworth RF. Amantadine treatment for Parkinson's disease during COVID-19: bimodal action targeting viral replication and the NMDA receptor. J Parkinsons Dis Alzheimer Dis. 2020;7(1):4.

26. Park MH, Kwon DY. A retrospective review of memantine use and COVID-19-associated mortality from a national database. J Med Virol. 2021;93(1):168-9. https://doi.org/10.1002/jmv.26266 (Epub 2020 Jul 14).

27. Butterworth RF. Amantadine for the treatment of traumatic brain injury and its associated cognitive and neurobehavioral complications. J Pharmacol Pharm Res. 2020;3(1):1-5.

28. Brison E, Jacomy H, Desforges M, Talbot PJ. Novel treatment with neuroprotective and antiviral properties against a neuroinvasive human respiratory virus. J Virol. 2014;88(3):1548-63. https://doi. org/10.1128/jvi.02972-13 (Epub 2013 Nov 13).

29. Kolocouris N, Foscolos GB, Kolocouris A, Marakos P, Pouli N, Fytas G, et al. Synthesis and antiviral activity evaluation of some aminoadamantane derivatives. J Med Chem. 1994;37(18):2896902. https://doi.org/10.1021/jm00044a010.

30. Kolocouris N, Kolocouris A, Foscolos GB, Fytas G, Neyts J, Padalko E, et al. Synthesis and antiviral activity evaluation of some new aminoadamantane derivatives. 2. J Med Chem. 1996;39(17):3307-18. https://doi.org/10.1021/jm950891z.

31. Chen F, Chan KH, Jiang Y, Kao RY, Lu HT, Fan KW, et al. In vitro susceptibility of 10 clinical isolates of SARS coronavirus to selected antiviral compounds. J Clin Virol. 2004;31(1):69-75. https://doi.org/10.1016/j.jcv.2004.03.003.

32. Cortés-Borra A, Aranda-Abreu GE. Amantadine in the prevention of clinical symptoms caused by SARS-CoV-2. Pharmacol Rep. 2021;18:1-4. https://doi.org/10.1007/s43440-021-00231-5 (Epub ahead of print). 